\title{
Antisense- and siRNA-mediated inhibition of the anti-apoptotic gene Bcl-xL for chemosensitization of bladder cancer cells
}

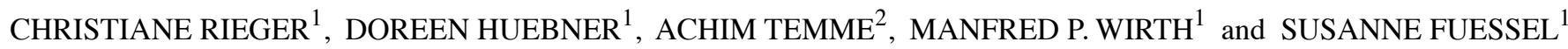 \\ Departments of ${ }^{1}$ Urology and ${ }^{2}$ Neurosurgery, Technische Universität Dresden, D-01307 Dresden, Germany
}

Received April 29, 2015; Accepted June 8, 2015

DOI: 10.3892/ijo.2015.3096

\begin{abstract}
Bcl-xL is an apoptosis inhibitor that is upregulated in bladder cancer $(\mathrm{BCa})$ and provides an attractive target for molecular therapies. Treatment with specific antisense oligodeoxynucleotides (AS-ODNs) or small interfering RNAs (siRNAs) were able to sensitize BCa cells to conventional chemotherapeutics. Ten new Bcl-xL-targeting AS-ODNs were systematically designed by using predicting software. AS-BX2034 and AS-BX2100 as well as the previously optimized siRNA construct si-BX713 were selected for further detailed in vitro analysis in the BCa cell lines UM-UC-3 and EJ28. Bcl-xL mRNA and protein expression levels, cell viability and apoptosis were examined $72 \mathrm{~h}$ after transfection. A single treatment with AS-BX2034 or AS-BX2100 caused only a low inhibition of the Bcl-xL mRNA expression with the highest reduction of $\leq 20 \%$ in UM-UC-3 cells. In contrast, a single treatment with si-BX713 strongly decreased Bcl-xL mRNA expression level by $\leq 69 \%$ in UM-UC-3 cells and by $\leq 86 \%$ in EJ28 cells. Both gene expression inhibitor types induced a low to moderate reduction of viability. Depending on the cell line, a combined treatment with AS-BX2100 or si-BX713 and cisplatin (CDDP) caused an additional inhibition of cell viability by $\sim 33$ and $38 \%$, respectively, compared to the respective control construct combined with CDDP. In comparison to the respective control treatment, combinations of AS-BX2100 and CDDP led to a stronger induction of apop-
\end{abstract}

Correspondence to: Christiane Rieger, Department of Urology, Technische Universität Dresden, Fetscherstrasse 74, D-01307 Dresden, Germany

E-mail: christiane.rieger@uniklinikum-dresden.de

Abbreviations: AS-ODN, antisense oligodeoxynucleotides; BAX, BCL-2-associated X protein; BAK, BCL-2 antagonist/killer; $\mathrm{BCa}$, bladder cancer; Bcl-xL, BCL2-like 1; BCL-2, B-cell CLL/lymphoma 2; CDDP, cisplatin; MIBC, muscle invasive bladder cancer; NMIBC, non-muscle invasive bladder cancer; PBS, phosphate-buffered saline; PI, propidium iodide; qPCR, quantitative PCR; SEM, standard error of the mean; siRNA, small interfering RNA; ss, single-stranded; TBP, TATA box binding protein; TUR, transurethral resection

Key words: antisense oligodesoxynucleotide, apoptosis, BCL2-like 1, cisplatin, small interfering RNA, transitional cell carcinoma tosis by $57 \%$ in UM-UC-3 cells and $44 \%$ in EJ 28 cells, whereas the combination of si-BX713 and CDDP enhanced apoptosis by 38 and $118 \%$ in UM-UC-3 and EJ 28 cells, respectively. Our comparative studies showed a stronger knockdown of Bcl-xL by the siRNA construct compared to AS-ODN treatment in both BCa cell lines. In combinatory treatments, the Bcl-xLdirected siRNA markedly enhanced the anti-proliferative and apoptotic effects of CDDP and therefore, may serve as suitable tool for chemosensitization of BCa cells.

\section{Introduction}

Apoptosis is one of the major mechanisms of cell death in response to cancer therapy (1). The regulatory balance of apoptosis is set by interactions on the outer mitochondrial membrane between members of three distinct subgroups of the BCL-2 (B-cell CLL/lymphoma 2) protein family: i) the BH3-only proteins, which mediate signals to initiate apoptosis, ii) the pro-apoptotic effector proteins BAX (BCL-2-associated X protein) and BAK (BCL-2 antagonist/ killer) and iii) the pro-survival family members such as BCL-2 and Bcl-xL (BCL2-like 1) (2). Bcl-xL is upregulated in a broad range of human cancers including bladder cancer $(\mathrm{BCa})(3,4)$. An overexpression of anti-apoptotic proteins such as Bcl-xL in $\mathrm{BCa}$ and other tumor entities is associated with disease maintenance and progression, resistance to chemotherapy, and poor clinical outcome (5).

$\mathrm{BCa}$ is the most common malignancy of the urinary tract and the seventh most prevalent cancer worldwide (6). At first diagnosis, $75 \%$ of the $\mathrm{BCa}$ are non-muscle invasive bladder cancers (NMIBC), which can be managed with transuretheral resection (TUR) by removing all visible lesions followed by an intravesical therapy (7). Recurrence rates in patients with NMIBC range from 31 to $78 \%$ within 5 years from diagnosis in the low-risk and high-risk subgroups, respectively (8). The remaining $25 \%$ of the $\mathrm{BCa}$ grow invasively and are grouped as muscle-invasive bladder cancers (MIBC). The standard treatment for locally advanced tumors is a radical cystectomy combined with a cisplatin-based chemotherapy where necessary (7). However, the risk of recurrence and progression remains considerable and requires additional and improved therapy strategies.

Resistance against chemotherapeutics such as cisplatin (cis-diamminedichloroplatinum (II); CDDP) is a major problem in the treatment of $\mathrm{BCa}$ and is caused by the upregulation of 
anti-apoptotic genes such as Bcl-xL $(9,10)$. Therefore, the knockdown of such genes by nucleic acid-based expression inhibitors could potentially sensitize BCa cells towards chemotherapy (10-12). Antisense oligodeoxynucleotides (AS-ODNs), which depend on ribonuclease $\mathrm{H}$-mediated cleavage of the mRNA, as well as RNA interference triggered by small interfering RNA molecules (siRNAs) act in a sequence-specific manner and can cause efficient knockdown of specific target genes $(12,13)$. It has previously been shown that AS-ODNs $(14,15)$ or siRNAs (16) directed at Bcl-xL can cause an efficient downregulation of Bcl-xL expression in different tumor models. Furthermore, Lebedeva et al demonstrated that a stable overexpression of $\mathrm{Bcl}-\mathrm{xL}$ in T24 BCa cells desensitized these cells to different cytotoxic agents and a subsequent AS-ODN-mediated Bcl-xL inhibition improved chemotherapy effectiveness (11).

However, the described Bcl-xL-directed AS-ODNs alone did not promote a significant inhibition of viability of $\mathrm{BCa}$ cells $(11,17,18)$. For this reason, alternative AS-ODNs with a potentially higher inhibitory efficiency would be preferable. Their therapeutic potential should be evaluated in comparison to siRNAs, which represent an expression inhibitor type with assumed higher and more specific inhibitory effects (12). Therefore, the aim of this study was to sensitize BCa cells to the commonly used chemotherapeutic CDDP in order to increase its cytotoxic efficacy by a specific pretreatment with AS-ODNs or siRNA constructs directed at the Bcl-xL mRNA. For this purpose we systematically designed new AS-ODNs for Bcl-xL knock-down in the BCa cell line UM-UC-3 representing an NMBIC cellular model and in the BCa cell line EJ28 originating from a MIBC and compared their effects to a previously characterized siRNA (16). Analyses of target expression, cell viability and apoptosis should reveal the effectiveness of the Bcl-xL downregulation by these expression inhibitors with regard to a chemosensitization of $\mathrm{BCa}$ cells.

\section{Materials and methods}

Design and selection of $A S-O D N$ and siRNA sequences. The secondary structure of the Bcl-xL mRNA (accession no. NM_138578) was predicted using the mfold 3.6 software (http://mfold.rna.albany.edu/?q=mfold). The ten most probable and stable structures with the lowest free energies $(\Delta \mathrm{G})$ were calculated and screened for potential single-stranded (ss) regions with at least six unpaired nucleotides. The conservation of the ss-motifs was calculated as percentage. Putative sites with a conservation of $\geq 40 \%$ were used as target sequences for the AS-ODN design (Table I). In addition, the software sfold 2.2 (http://sfold.wadsworth.org/cgi-bin/index.pl) generated a probability profile with predicted accessible sites for AS-ODN hybridization on the target mRNA. Compared to the sequences predicted with mfold the best conserved motifs were selected. Furthermore, AS-ODNs were selected based on a low binding energy, a GC content between 40 and $60 \%$ and the avoidance of GGGG motifs. A BLAST database search (http://blast.ncbi.nlm.nih.gov/Blast.cgi) was performed to exclude homologies to human coding RNA sequences. The eight finally selected nucleotide sequences of the AS-ODNs are shown in Table I.

AS-ODNs were synthesized as unmodified 20mers by biomers.net (Ulm, Germany). Two additional Bcl-xL-directed
AS-ODNs (20mers) were taken from the literature: 5'-AAAGT ATCCCAGCCGCCGTT-3' (17) and 5'-TCCCGGTTGCTCTG AGACAT-3' (ASO 15999) (15). The nonsense-ODN NS-K1 (5'-TAAGCTGTTCTATGTGTT-3') served as control-ODN for normalization (19). For siRNA treatment the previously characterized construct si-BX713 (5'-GGGACAGCAUAUCA GAGCU-3') was selected (16). The non-silencing-siRNA construct ns-si (reference: SR-CL000-005) from Eurogentec (Liège, Belgium) served as control.

Cell culture and treatment procedures. The human BCa cell line UM-UC-3 (ATCC, Rockville, MD, USA) was cultured in minimum essential medium with $10 \%$ fetal calf serum and $1 \%$ non-essential amino acids (all from Life Technologies, Karlsruhe, Germany). The human BCa cell line EJ28 (University of Frankfurt, Frankfurt, Germany) was cultured in Dulbecco's modified Eagle's medium with $4.5 \mathrm{~g} / 1$ glucose, $10 \%$ fetal calf serum and $1 \%$ non-essential amino acids (all from Life Technologies). Cells were cultured at $37^{\circ} \mathrm{C}$ in humidified atmosphere containing $5 \% \mathrm{CO}_{2}$.

For viability assays, 2,000 UM-UC-3 cells and 1,500 EJ28 cells were seeded per well into 96-well plates. For gene expression analysis, western blotting and apoptosis detection 150,000 UM-UC-3 cells and 15,000 EJ28 cells were seeded per well into 6-well plates. Seventy-two hours after seeding, cells were washed with phosphate-buffered saline (PBS) and transfected with the AS-ODNs (250 or $500 \mathrm{nM})$ and siRNAs $(40 \mathrm{nM})$ as well as with the corresponding controls using the liposomal transfection reagent DOTAP (Roche, Mannheim, Germany) at a 1:3 (w/w) ratio. The transfections were performed for $4 \mathrm{~h}$ in serum-free OptiMEM medium (Life Technologies). After transfection the medium was replaced by fresh culture medium. Cells were harvested from 6 -well plates by trypsin treatment $\left(0.05 \%\right.$ trypsin $/ 0.02 \%$ EDTA, $\left.5 \mathrm{~min}, 37^{\circ} \mathrm{C}\right) 24 \mathrm{~h}$ after start of transfection. For further analyses, detached and adherent cells were pooled, counted and analyzed together.

Furthermore, a CDDP concentration treatment series on UM-UC-3 and EJ28 cells should reveal the $\mathrm{IC}_{50}$ values and optimal CDDP concentrations for the chemosensitization experiments. According to the dose response curves CDDP concentrations of 0.25 and $0.5 \mu \mathrm{g} / \mathrm{ml}$ were used for UM-UC-3 cells and of 0.75 and $1.00 \mu \mathrm{g} / \mathrm{ml}$ for EJ28 cells, respectively. For chemosensitization experiments, $\mathrm{BCa}$ cells were first transfected with AS-ODNs (500 nM) or siRNAs (40 nM). Twenty-four hours after transfection start, BCa cells were treated with CDDP for another $24 \mathrm{~h}$. Subsequently, cells were washed with PBS and cultivated with fresh culture medium for another $24 \mathrm{~h}$. Analyses were performed $72 \mathrm{~h}$ after initiation of the transfection.

Expression analysis at RNA and protein level. Total RNA was isolated from $\leq 5 \times 10^{6}$ cells using the InviTrap Spin Cell RNA Mini kit according to the manufacturer's instructions (Invitek, Berlin, Germany). SuperScript II Reverse Transcriptase (Life Technologies) and random hexamer primers (Amersham Biosciences, Freiburg, Germany) were used for the reverse transcription of $500 \mathrm{ng}$ total RNA into first-strand cDNA according to the manufacturer's instructions. Transcript amounts of Bcl-xL and the reference gene TATA box binding protein (TBP) were determined by quantitative PCR (qPCR) 


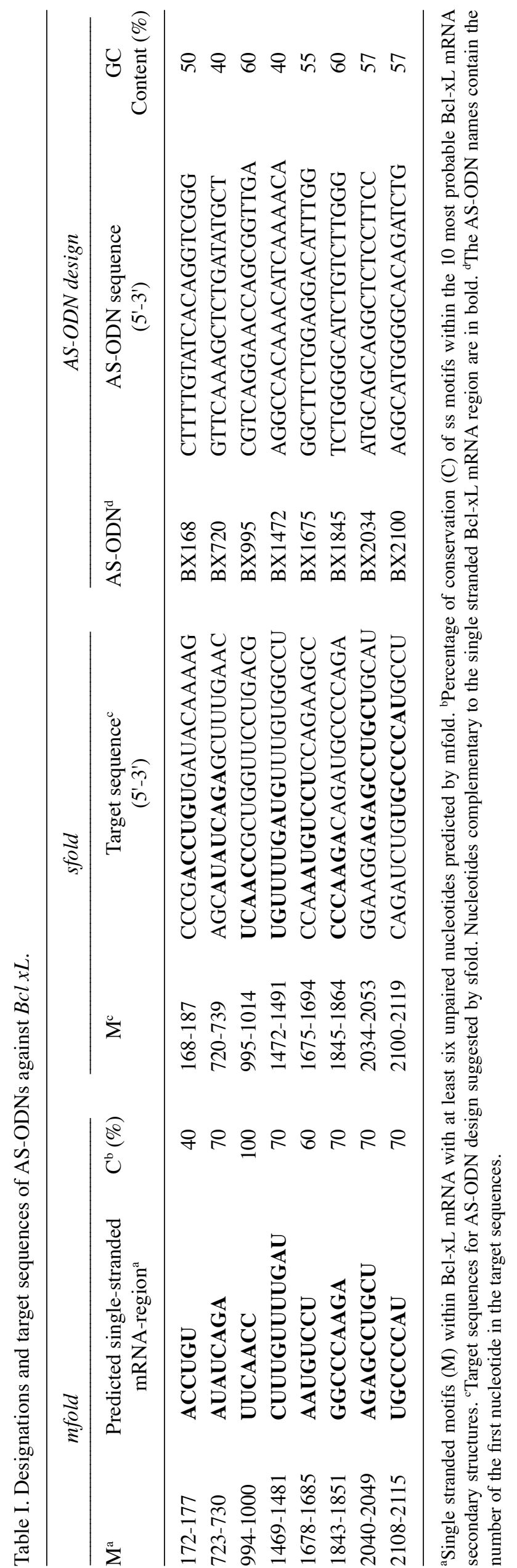

using the LightCycler system (Roche). For Bcl-xL mRNA quantitation a target-specific real-time reagent mix from AJ Roboscreen (Leipzig, Germany) containing the appropriate primers and a TaqMan probe was applied. For TBP the forward primer 5'-GAATATAATCCCAAGCGGTTTG-3', the reverse primer 5'-TTCACATCACAGCTCCCC-3' and hybridization probes labeled with fluorescein (5'-TTTCCCAGAACTGA AAATCAGTGCC-FL-3') and LC Red640 (5'-LC-TGGTTCG TGGCTCTCTTATCCTCATG-PH-3') were used. The relative transcript levels of Bcl-xL normalized to TBP were used for statistical calculations.

For western blot analysis $5 \times 10^{4}-1 \times 10^{5}$ cells per sample were lysed in loading buffer, incubated at $95^{\circ} \mathrm{C}$ for $5 \mathrm{~min}$ and separated on a $4-12 \%$ sodium dodecyl sulfate-polyacrylamide gel. Western blot analysis was performed according to a standard protocol using a monoclonal mouse anti-human Bcl-xL antibody (clone 2D1, OriGene Technologies, Rockville, MD, USA) at a 1:100 dilution. $\beta$-actin detected by a monoclonal mouse anti-human $\beta$-actin antibody (AC-74, Sigma-Aldrich, Steinheim, Germany) at a 1:50,000 dilution served as a loading control. As secondary antibody a polyclonal rabbit anti-mouse antibody conjugated with horseradish peroxidase (Dako, Glostrup, Denmark) was used and an enhanced chemiluminescence kit (Life Technologies) was employed for visualization.

Viability assays. Viability of UM-UC-3 cells was examined by using crystal violet, which stained the DNA of intact cells. For this test cells were fixed with $100 \mu 1$ methanol (Merck KGaA, Darmstadt, Germany) per well for $10 \mathrm{~min}$. Cells were washed with $100 \mu \mathrm{l}$ water and were subsequently stained with $100 \mu \mathrm{l}$ of a $0.1 \%$ crystal violet solution (AppliChem GmbH, Darmstadt, Germany) in 100\% ethanol (VWR, Darmstadt, Germany) for $10 \mathrm{~min}$. Afterwards, crystal violet was solubilized in $100 \mu \mathrm{l}$ $0.1 \mathrm{M}$ sodium citrate (Promega, Mannheim, Germany) and the absorbance was measured at $590 \mathrm{~nm}$ by using the Mithras LB 940 Multimode Microplate Reader (Berthold Technologies, Bad Wildbad, Germany). For the BCa cell line EJ28, cellular viability was analyzed using the cell proliferation reagent WST-1 (Roche). WST-1 reagent (10 $\mu \mathrm{l}$ per well) was added to the cells $72 \mathrm{~h}$ after start of transfection. Color development was monitored for up to $2 \mathrm{~h}$ by measuring the absorbance at 450 and $620 \mathrm{~nm}$ (reference). Furthermore, an alternative assay system based on the activity of a dead cell protease (Promega) was used according to the manufacturer's instructions for the determination of viability of both cell lines. All viability assays were performed in duplicates or triplicates. The results were normalized to the appropriate controls NS-K1 and ns-si.

Apoptosis detection. Apoptosis was assessed by Annexin V/ propidium iodide (PI) staining (Annexin V-FITC Apoptosis Detection Kit I; BD Biosciences, Heidelberg, Germany) 72 h after transfection start using flow cytometry (FACScan; BD Biosciences) according to the manufacturer's instructions. Annexin V staining and PI counterstaining allows the discrimination and quantification of early apoptotic cells (Annexin V-positive and PI-negative), late apoptotic cells (double-positive) and necrotic cells (Annexin V-negative and PI-positive). Annexin V-FITC/PI (FL1/FL2) plots of $2 \times 10^{4}$ cells were examined by quadrant analysis using the Flowing software 2.5.1 (http://www.flowingsoftware.com/). 


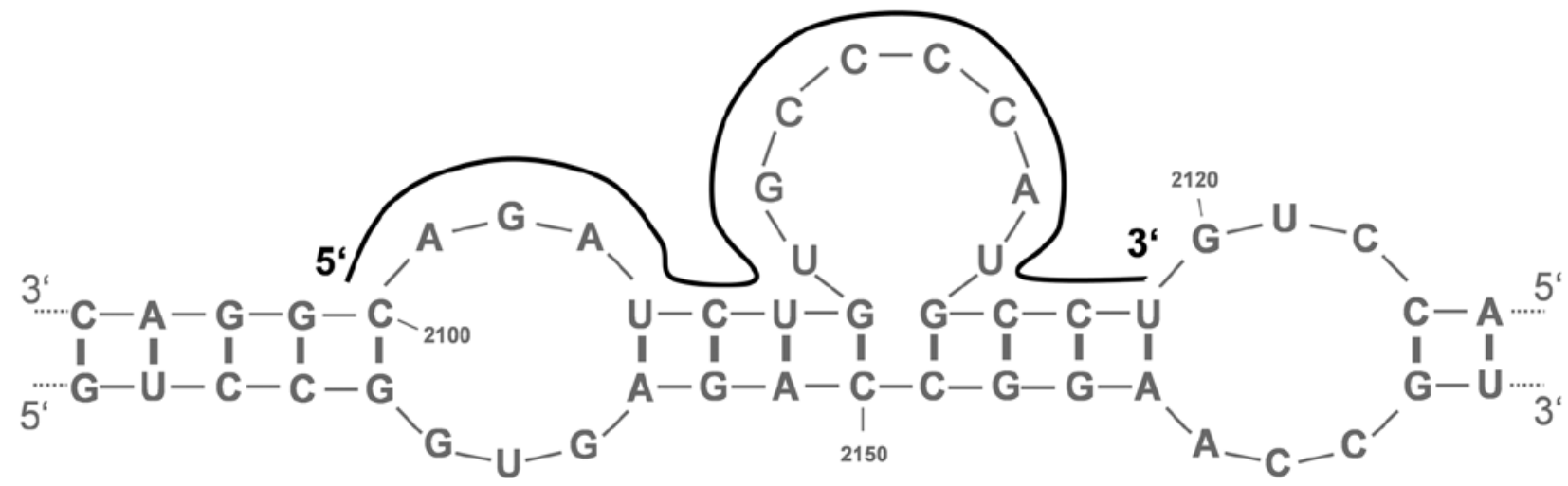

Figure 1. Binding region of AS-BX2100 at the Bcl-xL mRNA. The single-stranded AS-ODN AS-BX2100 (black line) is complementary to the target site between nucleotide positions 2100 and 2119 of Bcl-xL mRNA (accession no. NM138578). The numbers label the nucleotide positions within the Bcl-xL mRNA.

Statistics. All statistical calculations were performed using GraphPad Prism 5.03 (GraphPad Software, Inc., La Jolla, CA, USA). All results were presented as mean \pm standard error of the mean (SEM). Data from the treatment groups AS-ODN vs NS-K1, siRNA vs ns-si, CDDP vs untreated, AS-ODN+CDDP vs NS-K1+CDDP and siRNA+CDDP vs ns-si+CDDP were compared using ANOVA followed by Bonferroni's correction. A p-value $<0.05$ was considered as statistically significant.

Synergistic effects were calculated by the formula $X=[\mathrm{AB}] /$ $([\mathrm{A}]+[\mathrm{B}])$, where $[\mathrm{A}]$ is the single treatment with AS-ODN or siRNA and $[\mathrm{B}]$ the single treatment with CDDP. [AB] represents the combination treatment with AS-ODN or siRNA and CDDP. Synergy is defined as $X>1$, additivity is when $X=1$, and antagonism is when $\mathrm{X}<1$.

\section{Results}

Selection of AS-ODN and siRNA constructs in UM-UC-3 cells. The prediction of the secondary structure of the Bcl-xL mRNA using the mfold 3.6 software revealed the existence of several putative single-stranded regions. From ten theoretical and most stable mRNA structures, eight promising regions with a degree of conservation of $\geq 40 \%$ were selected for AS-ODN design. These target sequences in the Bcl-xL mRNA, which were also predicted by the sfold 2.2 software, were located at nucleotide positions 168-187 (BX168), 720-739 (BX720), 995-1014 (BX995), 1472-1491 (BX1472), 1675-1694 (BX1675), 1845-1864 (BX1845), 2034-2053 (BX2034) and 2100-2119 (BX2100) (Fig. 1 and Table I). BLAST database search revealed no homologies to other human mRNAs.

To select the best AS-ODN constructs for further studies, UM-UC-3 cells were transfected with $250 \mathrm{nM}$ of the newly designed AS-ODNs and two AS-ODNs from literature. AS-BX2100 showed the strongest inhibitory effect with a reduction of $19.8 \%$ of Bcl-xL mRNA expression in UM-UC-3 cells (Fig. 2). Therefore, this and another newly designed AS-ODN (AS-BX2034) were selected for further chemosensitization experiments. The si-BX713 construct caused a clear reduction of the Bcl-xL transcript levels by $76 \%$ in UM-UC-3 cells (data not shown).

Selection of CDDP concentrations for combined treatment. On the basis of the dose-response curves CDDP concentra-

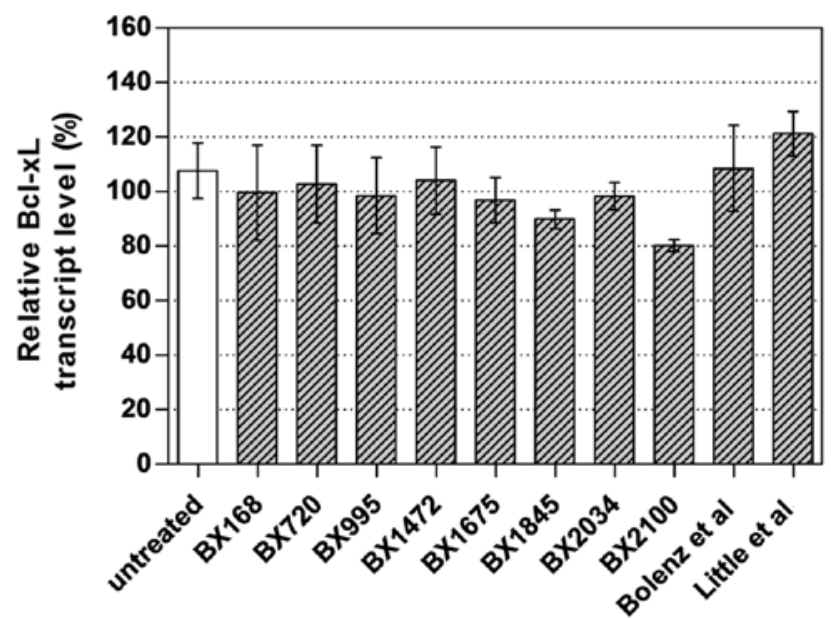

Figure 2. Evaluation of Bcl-xL mRNA level $24 \mathrm{~h}$ after start of AS-ODN transfection $(250 \mathrm{nM})$ in UM-UC-3 BCa cells. Relative Bcl-xL transcript levels (in relation to the reference gene TBP) were normalized to NS-K1 $(100 \%)$. Untreated cells served as control. Three independent experiments were performed and then averaged (mean \pm SEM). The results showed no statistically significant differences $(\mathrm{p}>0.05)$.

tions inducing only a moderate inhibition of cellular viability were selected. Ultimately, $0.25 \mu \mathrm{g} / \mathrm{ml}$ (CDDP1) and $0.5 \mu \mathrm{g} / \mathrm{ml}$ CDDP (CDDP2) where used for UM-UC-3 cells, whereas $0.75 \mu \mathrm{g} / \mathrm{ml}$ (CDDP1) and $1.00 \mu \mathrm{g} / \mathrm{ml}$ (CDDP2) were applied for EJ28 cells. Additionally, $\mathrm{IC}_{50}$ values of 1.49 and $3.23 \mu \mathrm{g} / \mathrm{ml}$ for CDDP were determined in UM-UC-3 and EJ28 cells, respectively (data not shown).

Effects on Bcl-xL mRNA and protein expression by single and combined treatment. The mRNA and protein expression levels of Bcl-xL in the BCa cell lines UM-UC-3 and EJ28 were evaluated $72 \mathrm{~h}$ after treatment with AS-ODNs or siRNAs with and without CDDP. A single treatment with the AS-BX2100 construct only reduced the mRNA expression level by $16 \%$ in UM-UC-3 cells and had no inhibitory effect on the Bcl-xL transcript level in EJ28 cells compared to NS-K1 (Fig. 3). In contrast, after treatment with si-BX713 a clear reduction of the Bcl-xL transcript levels by $69 \%$ in UM-UC-3 cells and by $86 \%$ in EJ28 cells, normalized to the ns-si control, was measured. Specific inhibition rates were detected by 
A

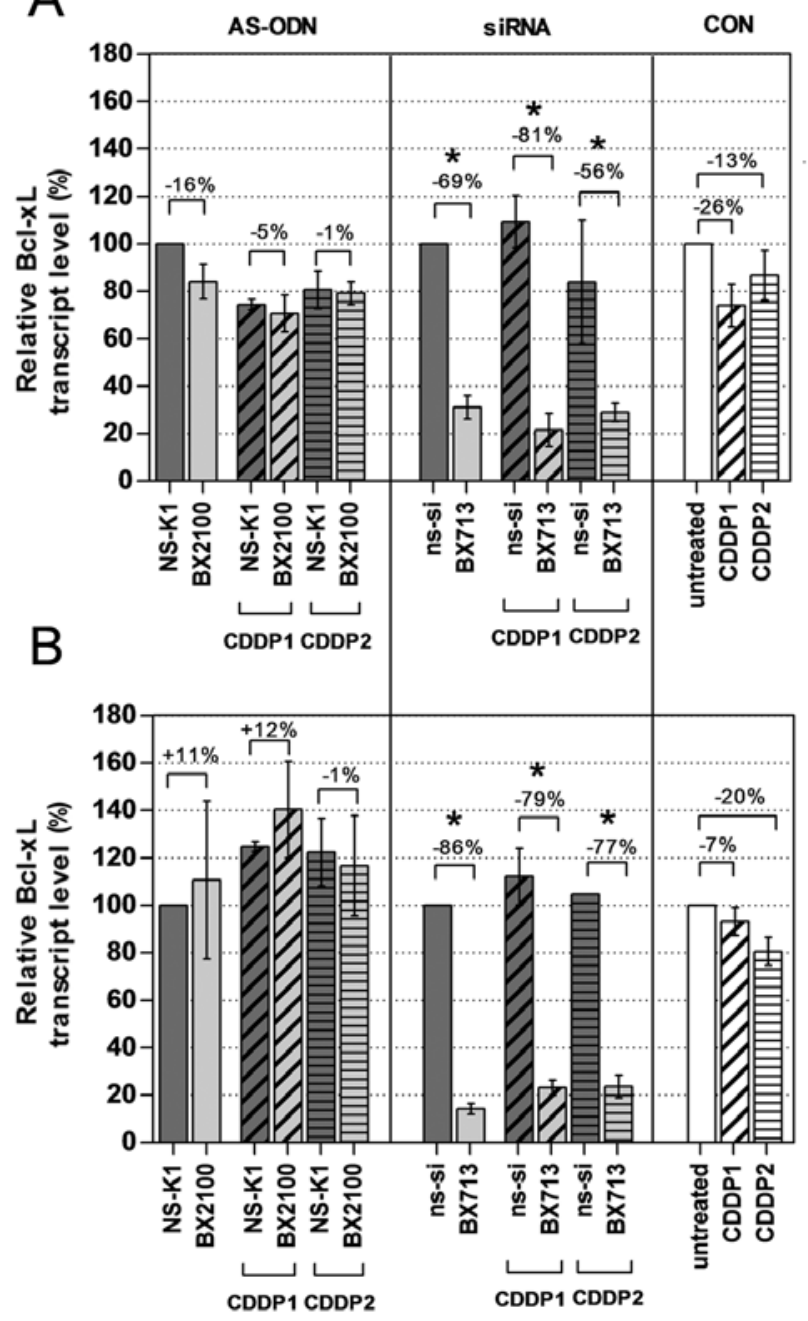

Figure 3. Relative Bcl-xL mRNA expression levels of UM-UC-3 (A) and EJ28 (B) cells $72 \mathrm{~h}$ after start of transfection with AS-ODNs (500 nM) and siRNAs $(40 \mathrm{nM})$ and combined treatment with CDDP. CDDP-concentrations for treatment of UM-UC-3 cells were $0.25 \mu \mathrm{g} / \mathrm{ml}$ (CDDP1) and $0.5 \mu \mathrm{g} / \mathrm{ml}$ (CDDP2). For EJ28 cells $0.75 \mu \mathrm{g} / \mathrm{ml}$ (CDDP1) and $1.00 \mu \mathrm{g} / \mathrm{ml}$ (CDDP2) were used. $\mathrm{Bcl}-\mathrm{xL}$ expression values were normalized to the reference gene TBP. Treatments with AS-ODNs or siRNAs were normalized to NS-K1 or ns-si, respectively (100\%). Single treatments with CDDP were normalized to untreated cells (100\%). Additional alterations (in perecentage, \%) and statistical significance are indicated above the bars $\left({ }^{*} \mathrm{p}<0.05\right)$. Two to three independent experiments were performed and then averaged (mean \pm SEM). CON, control.

normalizing the combination therapies of AS-BX2100+CDDP or si-BX713+CDDP to the appropriate control treatments NS-K1+CDDP or ns-si+CDDP, respectively. Treatments combining AS-BX2100 with CDDP1 or CDDP2 caused only little, or no effect on Bcl-xL expression in UM-UC-3 and EJ28 cells (Fig. 3). In contrast, a combined treatment with si-BX713 and CDDP caused a remarkable reduction of Bcl-xL transcript levels in both $\mathrm{BCa}$ cell lines. Inhibition rates were 81 and $56 \%$ (CDDP1/2) in UM-UC-3 cells and 79 and $77 \%$ (CDDP1/2) in EJ28 cells.

Western blot analysis showed that AS-BX2100 alone and in combination with CDDP only marginally reduced the Bcl-xL protein level in EJ28 cells compared to the respective control treatments (Fig. 4A). In contrast, a single treatment with si-BX713 as well as the combination with CDDP caused
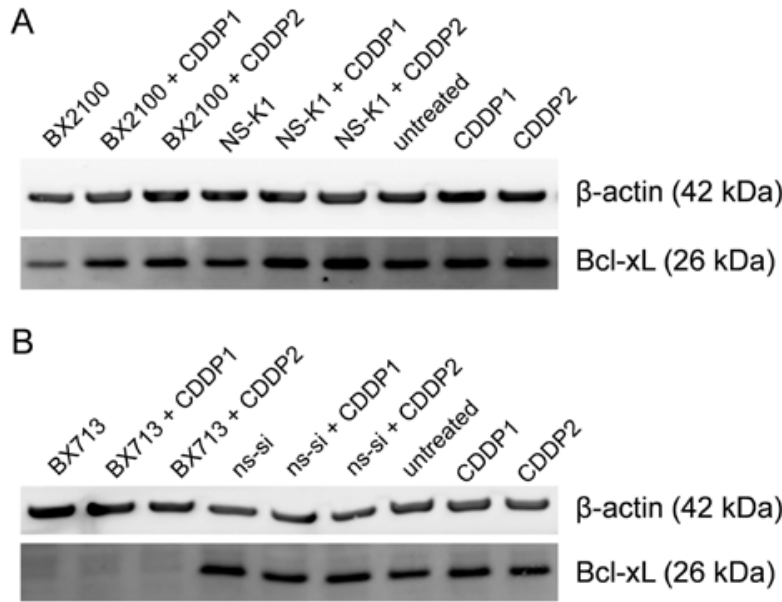

Figure 4. Bcl-xL protein expression levels of EJ28 cells $72 \mathrm{~h}$ after start of transfection with AS-ODNs (500 $\mathrm{nM})(\mathrm{A})$ and siRNAs $(40 \mathrm{nM})(\mathrm{B})$ and combined treatment with CDDP. For EJ28 cells $0.75 \mu \mathrm{g} / \mathrm{ml}$ (CDDP1) and $1.00 \mu \mathrm{g} / \mathrm{ml}$ (CDDP2) were used. $\beta$-actin served as loading control. Two independent experiments were performed and a representative western blot is depicted.

a complete inhibition of Bcl-xL protein expression compared to ns-si alone and ns-si+CDDP, respectively (Fig. 4B). In UM-UC-3 cells, no reduction at the Bcl-xL protein level following treatment with AS-BX2100 alone or in combination with CDDP was detectable (data not shown). However, the Bcl-xL protein level was moderately diminished after si-BX713 mono-treatment as well as combined treatment with CDDP (data not shown).

Effects on cellular viability by single and combined treatment. The inhibition of viability of the $\mathrm{BCa}$ cell lines was assessed by using crystal violet (UM-UC-3) and the WST-1 assay (EJ28) $72 \mathrm{~h}$ after start of transfection with AS-ODNs or siRNA with and without CDDP. Compared to the corresponding controls, a reduction of viability by 22,23 and $13 \%$ in UM-UC- 3 cells and by $0.5,5$ and $18 \%$ in EJ 28 cells by single treatments with AS-BX2034, AS-BX2100 and si-BX713, respectively, were observed (Fig. 5 and Table II). Treatments combining AS-ODNs with CDDP caused a moderate inhibition of cellular viability compared to NS-K1+CDDP in both cell lines. For example, a reduction of 33 and 13\% (CDDP1/2) in UM-UC-3 cells and 20 and 20\% (CDDP1/2) in EJ28 cells was observed after combinatory treatment with AS-BX2100. A combined treatment of si-BX713 and CDDP had only little or no effects in UM-UC-3 cells compared to ns-si+CDDP. In contrast, in EJ28 cells significant differences were observed for the treatment groups si-BX713+CDDP1 vs ns-si+CDDP1 and si-BX713+CDDP2 vs ns-si+CDDP2 with an additional inhibition of cellular viability of 33 and $38 \%$, respectively (Fig. 5 and Table II).

The combination of AS-BX2034, AS-BX2100 or si-BX713 with CDDP led to synergistic effects on viability mainly in EJ28 cells measured by WST-1 assay (Table II). Compared to the expected additive effects calculated from individual treatments the actual inhibition of cellular viability of EJ28 cells was increased by 2.5 -fold for the combination AS-BX2034+CDDP1, by 2.4-fold for the combination of 
Table II. Calculation of potential synergistic effects on cellular function of UM-UC-3 and EJ28 cells after AS-ODN/CDDP or siRNA/CDDP treatment. ${ }^{\mathrm{a}}$

\begin{tabular}{|c|c|c|c|c|c|c|}
\hline & \multicolumn{2}{|c|}{ Inhibition of viability ${ }^{\mathrm{b}}(\%)$} & \multicolumn{2}{|c|}{ Inhibition of viability ${ }^{\mathrm{c}}(\%)$} & \multicolumn{2}{|c|}{ Induction of apoptosis (\%) } \\
\hline & UM-UC-3 & EJ28 & UM-UC-3 & EJ28 & UM-UC-3 & EJ28 \\
\hline CDDP1 + NS-K1 & $5.2 \pm 4.9$ & $3.8 \pm 2.8$ & $0.4 \pm 5.2$ & $28.3 \pm 0.2$ & $29.3 \pm 23.3$ & $-21.0 \pm 3.1$ \\
\hline CDDP2 + NS-K1 & $30.7 \pm 13.0$ & $19.7 \pm 10.8$ & $26.5 \pm 1.7$ & $44.8 \pm 1.0$ & $37.7 \pm 53$ & $20.5 \pm 4.6$ \\
\hline AS-BX2034 alone & $21.6 \pm 3.8$ & $0.5 \pm 2.9$ & $-5.1 \pm 8.7$ & $2.6 \pm 0.5$ & ND & ND \\
\hline EE for CDDP1 & 26.7 & 4.4 & -4.7 & 30.9 & ND & ND \\
\hline ME for CDDP1 & $21.4 \pm 9.1$ & $10.9 \pm 4.3$ & $3.4 \pm 0.8$ & $31.5 \pm 4.0$ & ND & ND \\
\hline$n$-fold increase & 0.8 & 2.5 & -0.7 & 1.0 & ND & ND \\
\hline EE for CDDP2 & 52.3 & 20.2 & 24.1 & 47.4 & ND & ND \\
\hline ME for CDDP2 & $28.5 \pm 9.2$ & $19.2 \pm 8.7$ & $27.4 \pm 2.5$ & $46.0 \pm 5.9$ & ND & ND \\
\hline$n$-fold increase & 0.5 & 1.0 & 1.4 & 1.0 & ND & ND \\
\hline AS-BX2100 alone & $24.0 \pm 6.0$ & $5.7 \pm 9.7$ & $4.0 \pm 23.7$ & $32.6 \pm 1.1$ & $32.3 \pm 13.8$ & $26.1 \pm 0.4$ \\
\hline $\mathrm{EE}$ for CDDP1 & 29.1 & 9.6 & 21.4 & 60.8 & 61.5 & 5.1 \\
\hline ME for CDDP1 & $37.8 \pm 19.2$ & $22.8 \pm 14.7$ & $32.7 \pm 3.7$ & $57.2 \pm 3.1$ & $78.8 \pm 52.2$ & $13.7 \pm 34.8$ \\
\hline$n$-fold increase & 1.3 & 2.4 & 1.3 & 0.9 & 1.3 & 2.7 \\
\hline $\mathrm{EE}$ for $\mathrm{CDDP} 2$ & 54.7 & 25.4 & 50.2 & 77.3 & 69.9 & 64.7 \\
\hline ME for CDDP2 & $41.9 \pm 8.3$ & $35.5 \pm 11.8$ & $51.4 \pm 2.3$ & $72.4 \pm 1.8$ & $109.7 \pm 51.4$ & $59.7 \pm 32.5$ \\
\hline$n$-fold increase & 0.8 & 1.4 & 1.0 & 0.9 & 1.6 & 1.3 \\
\hline CDDP1 + ns-si & $11.9 \pm 3.7$ & $7.3 \pm 5.7$ & $15.4 \pm 1.8$ & $37.3 \pm 3.4$ & $58.2 \pm 50.1$ & $-15.8 \pm 2.4$ \\
\hline CDDP2 + ns-si & $33.4 \pm 8.8$ & $17.3 \pm 10.5$ & $32.2 \pm 0.1$ & $40.9 \pm 5.1$ & $45.3 \pm 31.3$ & $-6.2 \pm 15.8$ \\
\hline si-BX713 alone & $6.8 \pm 21.8$ & $18.6 \pm 11.3$ & $10.9 \pm 0.2$ & $29.9 \pm 6.3$ & $-8.7 \pm 17.8$ & $36.2 \pm 14.4$ \\
\hline EE for CDDP1 & 18.7 & 25.9 & 26.3 & 67.3 & 49.5 & 20.3 \\
\hline ME for CDDP1 & $17.3 \pm 18.7$ & $38.0 \pm 8.0$ & $35.8 \pm 1.7$ & $71.8 \pm 1.1$ & $52.1 \pm 55.9$ & $79.8 \pm 3.4$ \\
\hline$n$-fold increase & 0.9 & 1.5 & 1.4 & 1.1 & 1.1 & 3.9 \\
\hline $\mathrm{EE}$ for CDDP2 & 40.3 & 35.9 & 43.1 & 70.9 & 36.6 & 30.0 \\
\hline ME for CDDP2 & $30.1 \pm 10.2$ & $48.7 \pm 7.7$ & $62.8 \pm 1.3$ & $82.1 \pm 0.7$ & $94.1 \pm 37.2$ & $104.7 \pm 6.5$ \\
\hline$n$-fold increase & 0.7 & 1.4 & 1.5 & 1.2 & 2.6 & 3.5 \\
\hline
\end{tabular}

${ }^{a}$ CDDP1 and CDDP2 represent the different CDDP concentrations with CDDP1 $=0.25 \mu \mathrm{g} / \mathrm{ml}$ and CDDP2 $=0.5 \mu \mathrm{g} / \mathrm{ml}$ for UM-UC-3 and $\mathrm{CDDP} 1=0.75 \mu \mathrm{g} / \mathrm{ml}$ and CDDP2 $=1.00 \mu \mathrm{g} / \mathrm{ml}$ for EJ28. The expected effect (EE) is the additive inhibition of cellular viability or induction of apoptosis calculated from the single treatments. Measured effect (ME) is the actual inhibition of cellular viability or induction of apoptosis following treatment with AS-ODN or siRNA in combination with CDDP. $n$-fold increase of expected effect is calculated as the ratio of measured effect to expected effect. ${ }^{b}$ Viability was determined by using crystal violet and the WST-1 assay for UM-UC-3 and EJ28 cells, respectively. ${ }^{\mathrm{C}}$ Viability was determined by measuring the dead cell protease activity for both cell lines. ND, not determined; EE, expected effect; ME, measured effect.

AS-BX2100+CDDP1 and by 1.5-fold for the combination of si-BX713+CDDP1. Interestingly, the lower CDDP concentration led to stronger synergistic effects. In UM-UC-3 cells treatments combining AS-ODNs or siRNAs with CDDP caused no further increasing effect on viability measured by crystal violet (Table II).

Additional analysis of cellular viability based on the measurement of the dead cell protease activity should independently reveal the effectiveness of the AS-ODN and siRNA constructs $72 \mathrm{~h}$ after initiation of the transfection (Fig. 6). A single treatment with AS-BX2034 had no effect on cell viability in UM-UC-3 and EJ28 cells compared to NS-K1. In contrast, AS-BX2100 alone significantly reduced cell viability by 24 and $33 \%$ in UM-UC-3 and EJ 28 cells, respectively. A combined treatment of AS-BX2034 and CDDP caused no clear reduction of viability in the cell lines. In contrast, all combinations of AS-BX2100 with CDDP significantly reduced cell viability in both $\mathrm{BCa}$ cell lines whereupon the inhibition was generally stronger in EJ28 cells (Fig. 6). A single treatment with si-BX713 inhibited cell viability by 11 and $30 \%$ in UM-UC-3 and EJ28 cells, respectively. Inhibition rates of si-BX713 combined with CDDP were 24 and 45\% (CDDP1/2) in UM-UC-3 cells and 55 and 70\% (CDDP1/2) in EJ28 cells $($ all $\mathrm{p}<0.5)$. Compared to the added single effects the actual 


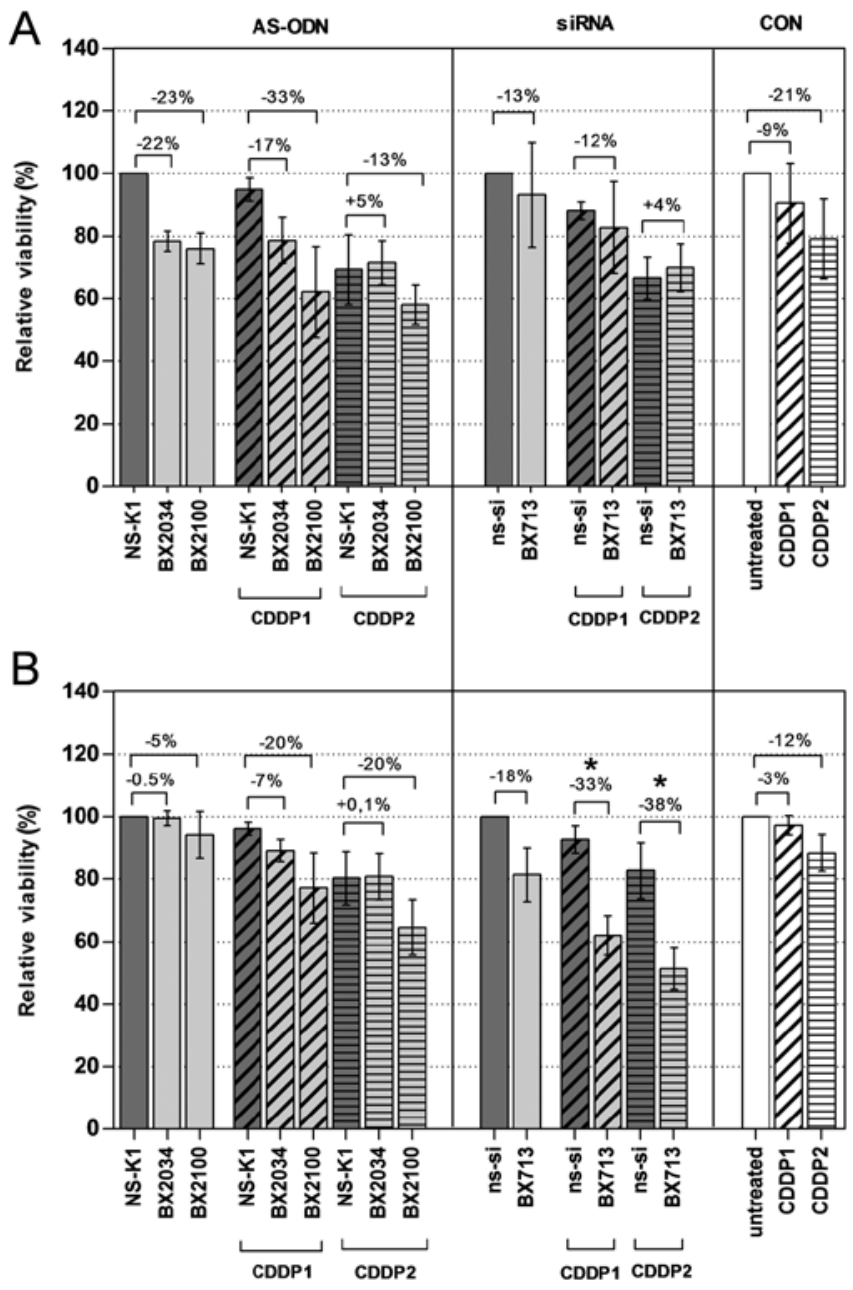

Figure 5. Relative viability of UM-UC-3 (A) and EJ28 (B) cells $72 \mathrm{~h}$ after the start of transfection with AS-ODNs $(500 \mathrm{nM})$ and siRNAs $(40 \mathrm{nM})$ and combined treatment with CDDP. Measurements were performed using crystal violet for UM-UC-3 cells and WST-1 viability assay for EJ28 cells. CDDP-concentrations for treatment of UM-UC-3 cells were $0.25 \mu \mathrm{g} / \mathrm{ml}$ (CDDP1) and $0.5 \mu \mathrm{g} / \mathrm{ml}$ (CDDP2). For EJ28 cells $0.75 \mu \mathrm{g} / \mathrm{ml}$ (CDDP1) and $1.00 \mu \mathrm{g} / \mathrm{ml}$ (CDDP2) were used. Treatments with AS-ODNs or siRNAs were normalized to NS-K1 or ns-si, respectively $(100 \%)$. Single treatments with CDDP were normalized to untreated cells (100\%). Additional alterations (in perecentage, \%) and statistical significance are indicated above the bars ( $\mathrm{p}<0.05)$. Two to three independent experiments were performed and then averaged (mean \pm SEM). CON, control.

inhibition of cellular viability in UM-UC-3 and EJ28 cells was synergistically increased by 1.5 - and 1.2 -fold for the combination of si-BX713+CDDP2 in UM-UC-3 and EJ28 cells, respectively (Table II).

Effects on apoptosis by single and combined treatment. Induction of apoptosis was measured $72 \mathrm{~h}$ after the start of the transfection in UM-UC-3 and EJ28 cells by an Annexin V-FITC/PI double staining. Rates of apoptosis were generally higher in EJ28 cells than in UM-UC-3 cells (Fig. 7). Treatment with AS-BX2100 with and without CDDP induced a moderate, but no significant increase in apoptosis by $26-57 \%$ normalized to the corresponding controls in both cell lines. This was also seen for siRNA treatment in UM-UC-3 cells. In contrast, a combined therapy with si-BX713 and CDDP produced a significant effect on apoptosis in EJ28 cells.
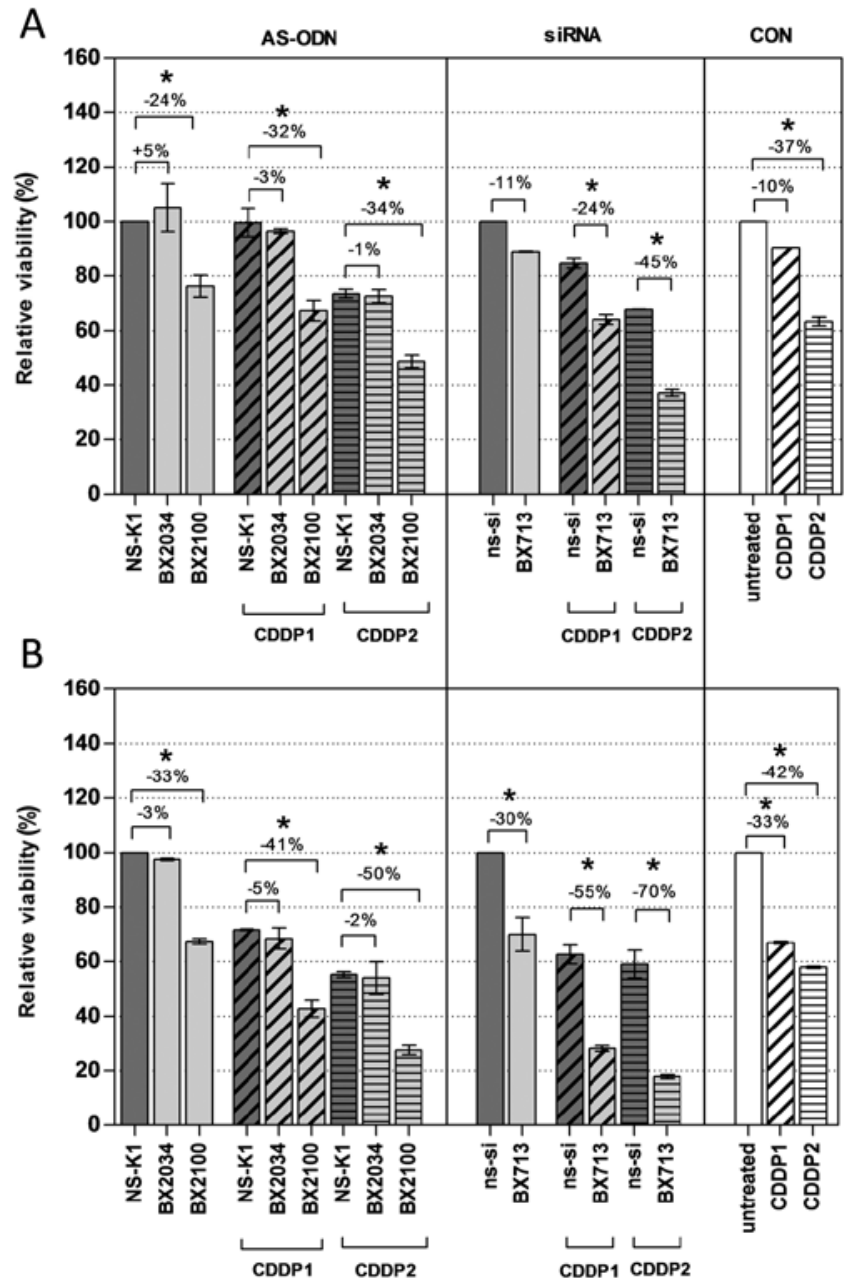

Figure 6. Relative activity of dead cell protease of UM-UC-3 (A) and EJ28 (B) cells $72 \mathrm{~h}$ after start of transfection with AS-ODNs $(500 \mathrm{nM})$ or siRNAs $(40 \mathrm{nM})$ and combined treatment with CDDP. Relative viability of UM-UC-3 and EJ28 cells were determined by measuring the dead cell protease activity. CDDP-concentrations for treatment of UM-UC-3 cells were $0.25 \mu \mathrm{g} / \mathrm{ml}$ (CDDP1) and $0.5 \mu \mathrm{g} / \mathrm{ml}$ (CDDP2). For EJ28 cells $0.75 \mu \mathrm{g} / \mathrm{ml}$ (CDDP1) and $1.00 \mu \mathrm{g} / \mathrm{ml}$ (CDDP2) were used. Treatments with AS-ODNs or siRNAs were normalized to NS-K1 or ns-si, respectively $(100 \%)$. Single treatments with CDDP were normalized to untreated cells (100\%). Additional alterations (in perecentage, \%) and statistical significance are indicated above the bars $(\mathrm{p}<0.05)$. Two to three independent experiments were performed and then averaged (mean \pm SEM). CON, control.

A single treatment with si-BX713 induced an increase of apoptosis by $49 \%$, whereas the combination with CDDP1 and CDDP2 led to a significant enhancement by 114 and $115 \%$, respectively, compared to the controls (Fig. 7). This observation was in agreement with the synergistic enhancement of 3.5- to 3.9-fold calculated for the combined treatments in EJ28 cells (Table II).

\section{Discussion}

One of the major clinical challenges in the successful treatment of tumors is the frequently occurring resistance to commonly applied therapies such as radiation or chemotherapy. One mechanism by which tumor cells develop resistance to cytotoxic agents is related to resistance to apoptosis (5). This is often a consequence of the increased 


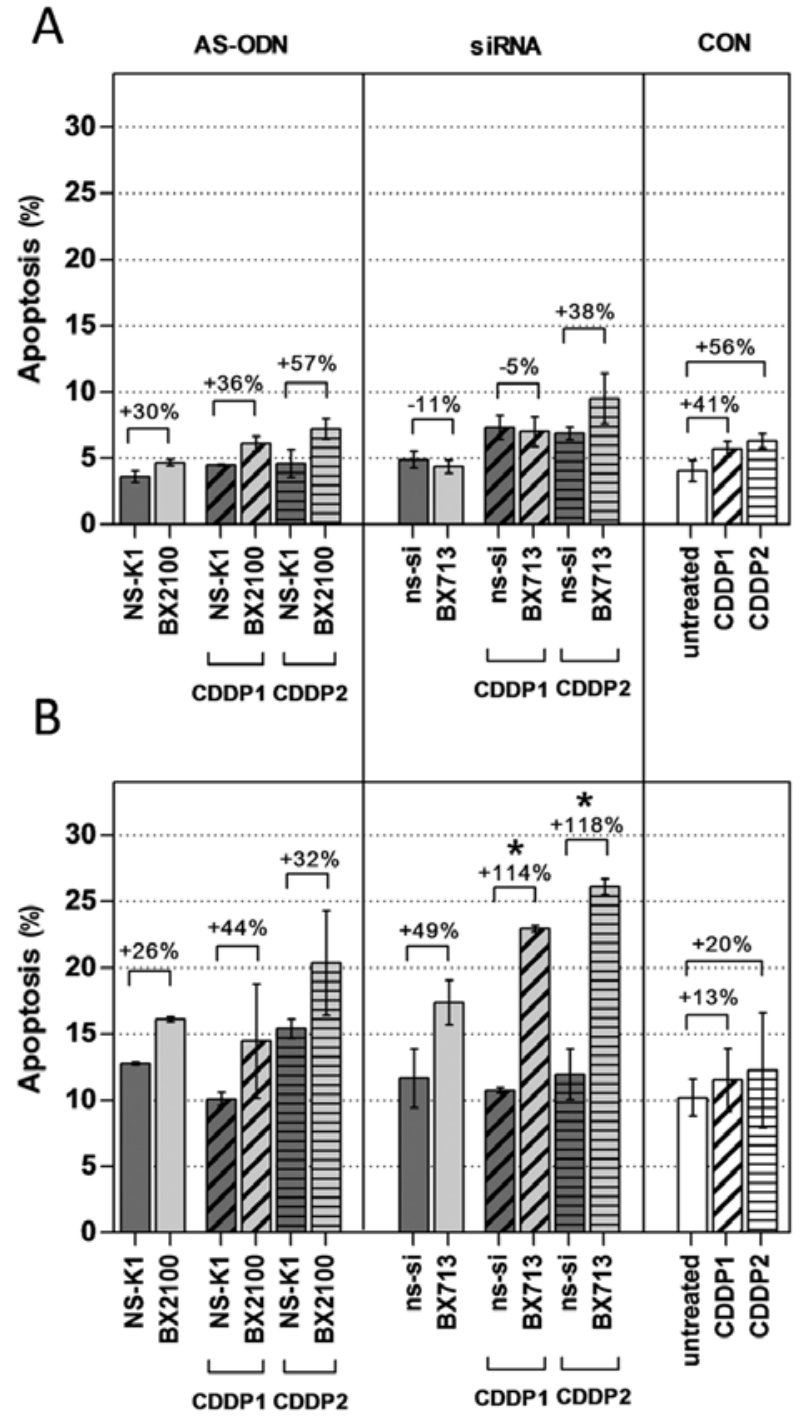

Figure 7. Percentage of apoptotic cells of UM-UC-3 (A) and EJ28 (B) cells $72 \mathrm{~h}$ after the start of the transfection with AS-ODNs $(500 \mathrm{nM})$ or siRNAs $(40 \mathrm{nM})$ and combined treatment with CDDP. CDDP-concentrations for treatment of UM-UC-3 cells were $0.25 \mu \mathrm{g} / \mathrm{ml}$ (CDDP1) and $0.5 \mu \mathrm{g} / \mathrm{ml}$ (CDDP2). For EJ28 cells $0.75 \mu \mathrm{g} / \mathrm{ml}$ (CDDP1) and $1.00 \mu \mathrm{g} / \mathrm{ml}$ (CDDP2) were used. Treatments with AS-ODNs or siRNAs were normalized to NS-K1 or ns-si, respectively $(100 \%)$. Single treatments with CDDP were normalized to untreated cells $(100 \%)$. Additional alterations (in perecentage, $\%$ ) and statistical significance are indicated above the bars $(\mathrm{p}<0.05)$. Two to three independent experiments were performed and then averaged (mean $\pm \mathrm{SEM}$ ). CON, control.

expression of anti-apoptotic proteins such as Bcl-xL $(11,18,20)$. Strategies to decrease the cellular expression of such proteins would enhance chemotherapy effectiveness (9). Therefore, the specific downregulation of these genes by nucleic acid-based inhibitors like AS-ODNs or siRNAs represents an attractive approach for molecular based sensitization $(11,21)$.

Lebedeva et al were able to show in vitro that a stable, forced overexpression of the anti-apoptotic protein $\mathrm{Bcl}-\mathrm{xL}$ in T24 BCa cells led to significant chemodesensitization (11). In contrast, the treatment of the $\mathrm{BCa}$ cell lines $\mathrm{T} 24$ and 5637 by AS-ODNs caused a clear downregulation of Bcl-xL mRNA and protein expression and increased their sensitivity to cytotoxic agents. Interestingly, an AS-ODN treatment in T24 and 5637 cells alone did not decrease cellular viability, indicating that $\mathrm{Bcl}-\mathrm{xL}$ might not be essential for the survival of these tumor cells but for the regulation of programmed cell death, which is a consequence of apoptotic stimuli (11). Furthermore, the inhibitory efficiency of AS-ODNs depended on the sequences and modifications of the constructs as well as on the type of delivery agent (11). However, it was reported in several studies that a treatment of BCa cells with Bcl-xLdirected AS-ODNs alone did not induce significant inhibition of viability, whereas a combination with chemotherapeutics promoted a significant increase of apoptosis in tumor cell lines as evidence for chemosensitization $(11,14,17)$. To improve the efficiency of Bcl-xL-targeted AS-ODNs we systematically designed and characterized new AS-ODNs.

For the design of AS-ODNs a combination of the prediction tools mfold and sfold was used, which can determine the most probable occurring secondary structure of target mRNAs with minimal overall free energy as a potential AS-ODN target site (22). Furthermore, the conservation of the ss-motifs, the achievement of an optimal CG content and a BLAST search for avoidance of unspecific hybridization was considered in contrast to former studies $(11,23)$. The AS-ODNs designed by this strategy were compared to the therapeutic potential of a Bcl-xL-directed siRNA which was reported to be efficient in previous studies (16).

The constructs AS-BX2100, AS-BX2034 and si-BX713 were selected for detailed analyses in the $\mathrm{BCa}$ cell lines UM-UC-3 and EJ28 to compare their efficacy in enhancing the cytotoxic effects of the chemotherapeutic agent CDDP. Analysis of changes in Bcl-xL expression in UM-UC-3 and EJ28 cells treated with si-BX713 with or without CDDP revealed a similar distinct downregulation of Bcl-xL mRNA expression by $69-86 \% 72 \mathrm{~h}$ after the start of the transfection. A single and combined treatment of both $\mathrm{BCa}$ cell lines with si-BX713 or si-BX713+CDDP caused a considerably higher reduction of Bcl-xL mRNA and protein expression (Figs. 3 and 4) compared to AS-BX2100 or AS-BX2100+CDDP, respectively. Moreover, the treatment of UM-UC-3 and EJ28 cells with si-BX713 caused a stronger reduction of cell viability as well as a more potent induction of apoptosis compared to an AS-ODN treatment in nearly all cases. In similar studies, Fuessel et al also demonstrated a higher efficiency of siRNAs compared to AS-ODNs directed at the anti-apoptotic survivin, which clearly reduced target expression and cell viability of EJ28 and 5637 BCa cells (24).

Numerous studies revealed siRNA constructs as superior tools with significantly lower IC50 values and prolonged effects in comparison to AS-compounds $(13,25,26)$. In our studies, si-BX713 showed better inhibition of Bcl-xL mRNA expression at a lower dosis (40 nM) compared to AS-BX2100 $(500 \mathrm{nM})$ (Fig. 3). Nevertheless, in our studies we used an even lower dose of AS-ODNs (500 $\mathrm{nM})$ compared to other studies that applied higher doses of AS-ODNs of up to $5 \mu \mathrm{M}$ $(14,18)$ which in turn could increase the risk of unspecific effects. Therefore, AS-ODNs and siRNAs must be used at the lowest effective concentration to minimize unwanted side activities. In general, there are several advantages of using siRNA constructs as inhibitors of gene expression $(13,27)$. The higher stability of siRNA constructs as well as their possible recycling after target mRNA degradation and thus, the reuse as catalytic compounds may explain the longer duration of 
action in comparison to AS-ODNs $(13,26)$. Off-target effects are the consequence of non-sequence-specific downregulation of gene expression. For example, the construct AS-BX2034 had no influence on the Bcl-xL mRNA expression level but on the viability of UM-UC-3 cells (Figs. 2 and 6). This might be caused by off-target effects. Nevertheless, for effective application of nucleic acid-based inhibitors some of the problems are similar: efficient delivery, enhanced stability, minimization of off-target effects and the identification of degradation-sensitive sites in the target RNAs (13).

In most of the tested combinations, the enhancement of CDDP effects with regard to inhibition of viability and induction of apoptosis by a pretreatment with AS-ODNs or siRNAs were stronger in EJ28 cells compared to UM-UC-3 cells. This difference might be caused by different basic Bcl-xL expression levels in these cell lines. The MIBC-derived cell line EJ28 displayed a 10-fold higher basic Bcl-xL mRNA expression level compared to the NMIBC-derived cell line UM-UC-3 (data not shown). Hence, it could be possible that an increased expression of Bcl-xL is associated with advanced $\mathrm{BCa}$ stages and more important for cell survival compared to those cell lines which express Bcl-xL at a lower level.

Interestingly, a stronger inhibition of viability was observed for lower CDDP doses (CDDP1) in combination with Bcl-xL downregulation by AS-BX2100 and AS-BX2034 in EJ28 cells (Fig. 5 and Table II). It is possible that a treatment with higher CDDP concentrations (CDDP2) alone could already reach strong effects on $\mathrm{BCa}$ cells and thus, no further inhibition of cell viability with an AS-ODN pretreatment could be achieved. An obvious advantage of lower doses of chemotherapeutic agents is a lower rate of adverse effects caused by drug toxicity in patients. In contrast, the pretreatment with the Bcl-xL-directed siRNA always induced synergistic effects compared to the single effects of both CDDP doses, possibly originating from its superior effectiveness and specificity.

In this study, a number of new AS-ODNs was systematically designed and characterized with regard to the inhibition of $\mathrm{Bcl}-\mathrm{xL}$ expression in two different $\mathrm{BCa}$ cell lines. The potential for chemosensitization of the most promising AS-constructs was compared to a specific siRNA, which induced a significantly stronger reduction of Bcl-xL expression at mRNA and protein levels. A combined treatment with siRNA and CDDP led to a more potent inhibition of cell viability as well as to a stronger induction of apoptosis compared to AS-BX2100 and CDDP in both cell lines. Therefore, Bcl-xL might serve as a suitable target depending on the basic expression levels in the tumor. The application of siRNAs as gene expression inhibitors and chemosensitizers appears more promising than AS-ODNs for the treatment of BCa.

\section{Acknowledgements}

This study was supported by the German Cancer Aid (grant no. 109616). Furthermore, the authors are grateful to Kati Erdmann for her useful advice.

\section{References}

1. Luo J, Solimini NL and Elledge SJ: Principles of cancer therapy: Oncogene and non-oncogene addiction. Cell 136: 823-837, 2009.
2. Czabotar PE, Lessene G, Strasser A and Adams JM: Control of apoptosis by the BCL-2 protein family: Implications for physiology and therapy. Nat Rev Mol Cell Biol 15: 49-63, 2014.

3. Yu X, Yang L, Cairns MJ, Dass C, Saravolac E, Li X and Sun LQ Chemosensitization of solid tumors by inhibition of Bcl-xL expression using DNAzyme. Oncotarget 5: 9039-9048, 2014.

4. Gazzaniga P, Gradilone A, Silvestri I, Gandini O, Giuliani L, Vincenzoni A, Gallucci M, Frati L and Agliano AM: Variable levels of bcl-2, bcl-x and bax mRNA in bladder cancer progression. Oncol Rep 5: 901-904, 1998.

5. Giménez-Bonafé P, Tortosa A and Pérez-Tomás R: Overcoming drug resistance by enhancing apoptosis of tumor cells. Curr Cancer Drug Targets 9: 320-340, 2009.

6. Youssef RF and Lotan Y: Predictors of outcome of non-muscleinvasive and muscle-invasive bladder cancer. Sci World J 11: 369-381, 2011.

7. Witjes JA, Compérat E, Cowan NC, De Santis M, Gakis G, Lebret T, Ribal MJ, Van der Heijden AG and Sherif A; European Association of Urology: EAU guidelines on muscle-invasive and metastatic bladder cancer: Summary of the 2013 guidelines. Eur Urol 65: 778-792, 2014.

8. Babjuk M, Burger M, Zigeuner R, Shariat SF, van Rhijn BW, Compérat E, Sylvester RJ, Kaasinen E, Böhle A, Palou Redorta J, et al; European Association of Urology: EAU guidelines on non-muscle-invasive urothelial carcinoma of the bladder: Update 2013. Eur Urol 64: 639-653, 2013.

9. Duggan BJ, Gray S, Johnston SR, Williamson K, Miyaki H and Gleave M: The role of antisense oligonucleotides in the treatment of bladder cancer. Urol Res 30: 137-147, 2002.

10. Yoshimine S, Kikuchi E, Kosaka T, Mikami S, Miyajima A, Okada Y and Oya M: Prognostic significance of Bcl-xL expression and efficacy of Bcl-xL targeting therapy in urothelial carcinoma. Br J Cancer 108: 2312-2320, 2013.

11. Lebedeva I, Raffo A, Rando R, Ojwang J, Cossum P and Stein CA: Chemosensitization of bladder carcinoma cells by bcl-xL antisense oligonucleotides. J Urol 166: 461-469, 2001.

12. Achenbach TV, Brunner B and Heermeier K: Oligonucleotidebased knockdown technologies: Antisense versus RNA interference. Chem Biochem 4: 928-935, 2003.

13. Scherer LJ and Rossi JJ: Approaches for the sequence-specific knockdown of mRNA. Nat Biotechnol 21: 1457-1465, 2003.

14. Bolenz C, Becker A, Trojan L, Schaaf A, Cao Y, Weiss C, Alken P and Michel MS: Optimizing chemotherapy for transitional cell carcinoma by application of bcl- 2 and bcl-xL antisense oligodeoxynucleotides. Urol Oncol 25: 476-482, 2007.

15. Littlejohn JE, Cao X, Miller SD, Ozvaran MK, Jupiter D, Zhang L, Rodarte C and Smythe WR: Bcl-xL antisense oligonucleotide and cisplatin combination therapy extends survival in SCID mice with established mesothelioma xenografts. Int J Cancer 123: 202-208, 2008.

16. Kunze D, Erdmann K, Froehner M, Wirth MP and Fuessel S: siRNA-mediated inhibition of antiapoptotic genes enhances chemotherapy efficacy in bladder cancer cells. Anticancer Res 32: 4313-4318, 2012

17. Bolenz C, Weiss C, Wenzel M, Gabriel U, Steidler A, Becker A, Herrmann E, Trojan L and Michel MS: In vivo evaluation of intravesical paclitaxel and combined bcl-xL antisense oligodeoxynucleotide treatment for orthotopic urothelial carcinoma. J Cancer Res Clin Oncol 135: 679-686, 2009.

18. Gabriel U, Bolenz C, Becker A, Schaaf A, Steidler A, Trojan L, Weiss $C$ and Michel MS: Evaluation of cytotoxic effects induced by bcl-2 and bcl-xL antisense-oligodeoxynucleotides in normal urothelium and transitional cell carcinoma. Oncol Rep 20: 1419-1423, 2008

19. Kraemer K, Fuessel S, Schmidt U, Kotzsch M, Schwenzer B, Wirth MP and Meye A: Antisense-mediated hTERT inhibition specifically reduces the growth of human bladder cancer cells. Clin Cancer Res 9: 3794-3800, 2003.

20. Kirsh EJ, Baunoch DA and Stadler WM: Expression of bcl-2 and bcl-X in bladder cancer. J Urol 159: 1348-1353, 1998.

21. Kunze D, Wuttig D, Fuessel S, Kraemer K, Kotzsch M, Meye A, Grimm MO, Hakenberg OW and Wirth MP: Multitarget siRNA inhibition of antiapoptotic genes (XIAP, BCL2, BCL-X(L)) in bladder cancer cells. Anticancer Res 28B: 2259-2263, 2008.

22. Chan JH, Lim S and Wong WS: Antisense oligonucleotides: From design to therapeutic application. Clin Exp Pharmacol Physiol 33: 533-540, 2006.

23. Lebedeva I, Rando R, Ojwang J, Cossum P and Stein CA: Bcl-xL in prostate cancer cells: Effects of overexpression and downregulation on chemosensitivity. Cancer Res 60: 6052-6060, 2000. 
24. Fuessel S, Herrmann J, Ning S, Kotzsch M,KraemerK, Schmidt U, Hakenberg OW, Wirth MP and Meye A: Chemosensitization of bladder cancer cells by survivin-directed antisense oligodeoxynucleotides and siRNA. Cancer Lett 232: 243-254, 2006.

25. Miyagishi M, Hayashi $M$ and Taira K: Comparison of the suppressive effects of antisense oligonucleotides and siRNAs directed against the same targets in mammalian cells. Antisense Nucleic Acid Drug Dev 13: 1-7, 2003.
26. Bertrand JR, Pottier M, Vekris A, Opolon P, Maksimenko A and Malvy C: Comparison of antisense oligonucleotides and siRNAs in cell culture and in vivo. Biochem Biophys Res Commun 296: $1000-1004,2002$.

27. Kretschmer-Kazemi Far R and Sczakiel G: The activity of siRNA in mammalian cells is related to structural target accessibility: A comparison with antisense oligonucleotides. Nucleic Acids Res 31: 4417-4424, 2003. 